\title{
An uncommon presentation of non- small-cell lung cancer with acrometastases to the great toe and index finger
}

\author{
Jamie Reynolds, PA-C, and Savitri P Skandan, MD
}

Department of Internal Medicine, Division of Hematology and Oncology, Lehigh Valley Hospital, Muhlenberg, Pennsylvania

A crometastases as initial presentation of metastatic cancer is an extremely rare finding. We describe an unusual case of late-stage non-small-cell lung cancer with metastatic lesions to the great toe and index finger with associated pain in those areas as the only presenting symptom.

\section{Case presentation and summary}

A 71-year-old white woman was referred to the emergency department by her primary care physician for necrosis and swelling of the left great toe for work-up of possible osteomyelitis (Figure 1). Before she presented to her physician, she had been complaining of severe pain, swelling, and erythema of the left great toe that had lasted for 1-2 months. Infection was initially suspected. She completed 2 courses of oral antibiotics with no improvement. She was also complaining of similar symptoms on the left index finger and attributed her symptoms to an injury a month earlier (Figure 2). The pain was so severe that she was not able to bear weight on her left foot. An outpatient X-ray of her left great toe raised her physician's concerns that it might be osteomyelitis so she was referred to the emergency department.

Her medical history revealed that she had a 40 pack-year tobacco history and had quit smoking about 5 years before presentation. She had been medically noncompliant and had not been to see any physician in more than 10 years, and therefore had no documented medical comorbidities. She had no history of recent unintentional weight loss, weakness, fatigue, hemoptysis, headaches, double vision, nausea, or vomiting. She was otherwise in her normal state of health.

In the emergency department, $\mathrm{X}$-rays were done of her left foot and left index finger. The results suggested possible osteomyelitis, but there was also con- cern for a lytic lesion with suspicion of metastasis. The patient was admitted for further evaluation and pain control. She had a magnetic-resonance imaging (MRI) scan of the left foot, which revealed an indeterminate expansile lesion of the distal phalanx of the great toe suggestive of possible metastatic disease (Figure 3). She was referred to the medical oncology department for a malignancy work-up.

The malignancy work-up included a computedtomography (CT) scan of the chest, abdomen, and pelvis; a nuclear bone scan; and an MRI of the brain. The CT scan of the chest revealed a mass in the right lower lobe of the lung of $5.5 \times 5.4 \mathrm{~cm}$, a subcarinal lymph node of $4.2 \times 3.0 \mathrm{~cm}$, a small indeterminate 10-mm hypodense lesion in the liver, and a lytic lesion at the T10 vertebral body (Figure 4). The MRI of the brain revealed 2 masses in the right frontal lobe of $3.6 \times 3.0 \mathrm{~cm}$ and $3.4 \times 3.7 \mathrm{~cm}$, a lesion in the left parietal lobe of $5.2 \times 3.8 \mathrm{~cm}$, a ventricular mass measuring $1.5 \times 1.4 \mathrm{~cm}$, vasogenic edema, and leptomeningeal and dural enhancement consistent with tumor spread (Figure 5).

The patient underwent a CT-guided biopsy of the liver mass, as well as a biopsy of left index finger to establish a diagnosis. Both of the lesions were consistent with metastatic adenocarcinoma. Immunoperoxidase stains were positive for CK7, TTF-1, MOC31, and mucicarmine; and negative for CK20, napsin A, Hep Par 1, and CA 19-9, which confirmed a diagnosis of adenocarcinoma of lung primary (Figures 6 and 7). The patient was then referred for evaluation to the radiation oncology, plastic surgery, and podiatry departments for treatment options for the metastatic digit lesions.

For local control of her pain, the patient underwent amputation the distal portion of left index finger and the left great toe. Postoperatively, she became almost completely free of pain and was able to walk

Accepted for publication June 22, 2015. Correspondence: Jamie Reynolds, PA-C; Jamie.Reynolds@lvhn.org. Disclosures: The authors report no disclosures or conflicts of interest. JCSO 2016;14:122-125. (C2016 Frontline Medical Communications. doi: 10.12788/jcso.0219. 


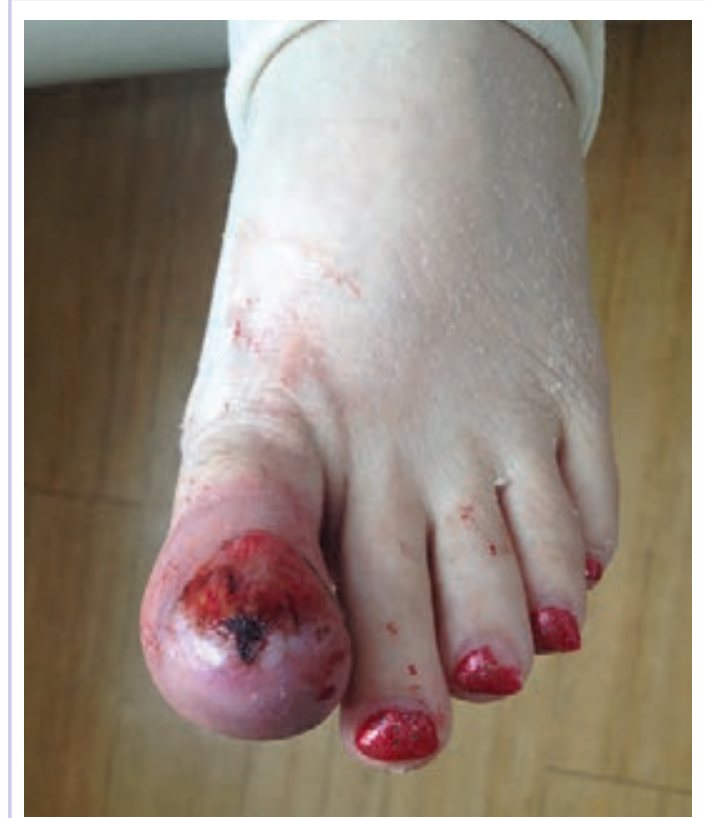

FIGURE 1 Left great toe involved with cancer causing swelling, erythema and necrosis at the distal end of digit. Photo provided by patient.

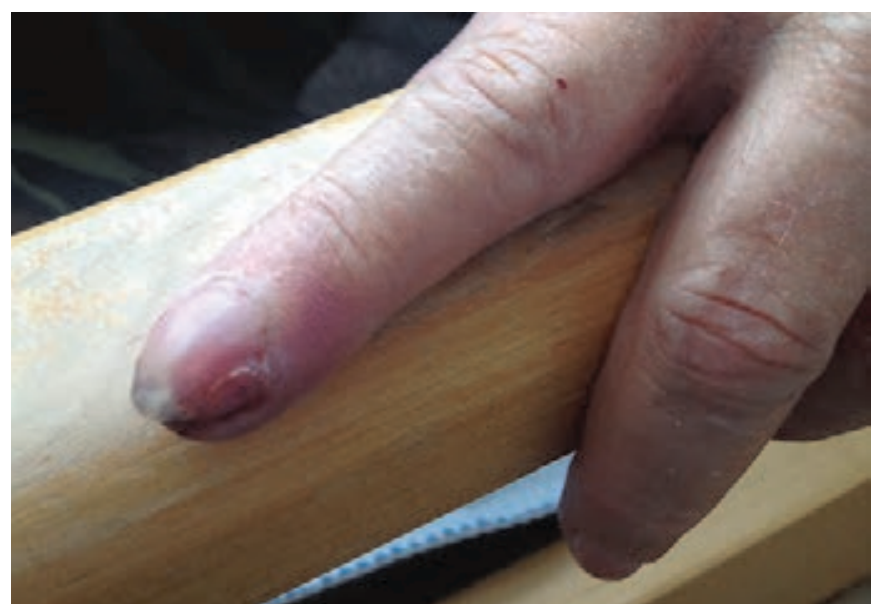

FIGURE 2 Left index finger involved with cancer causing swollen digit with erythema and necrosis. Photo provided by patient.

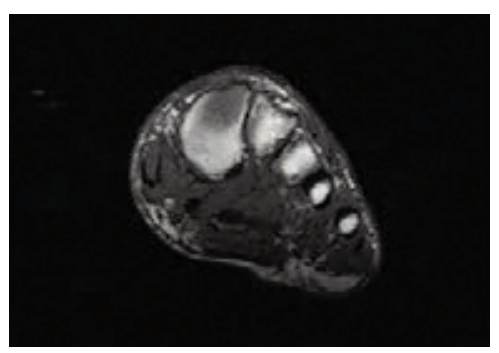

FIGURE 3 MRI of the patient's left foot showing osteolytic lesion in the great toe.



FIGURE 4 CT scan of the patient's chest showing a subcarinal lymph node of $4.2 \times 3.0 \mathrm{~cm}$

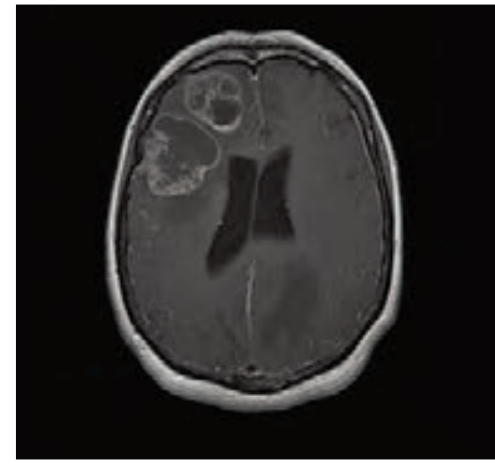

FIGURE 5 MRI of the patient's brain showing right frontal lobe masses with surrounding vasogenic edema.

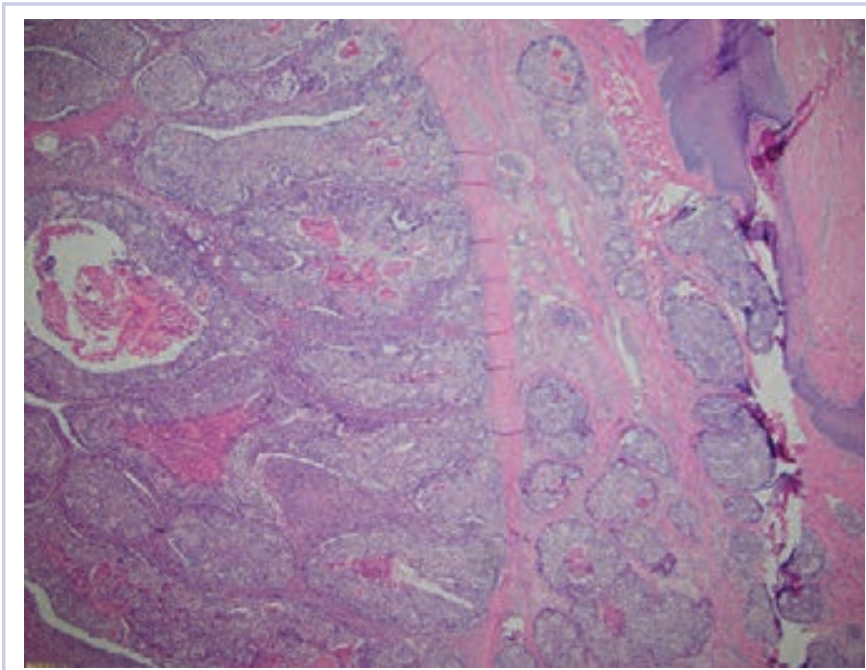

FIGURE 6 H\&E stain at 10x power showing metastatic adenocarcinoma on the underneath side of skin surface of left great toe.

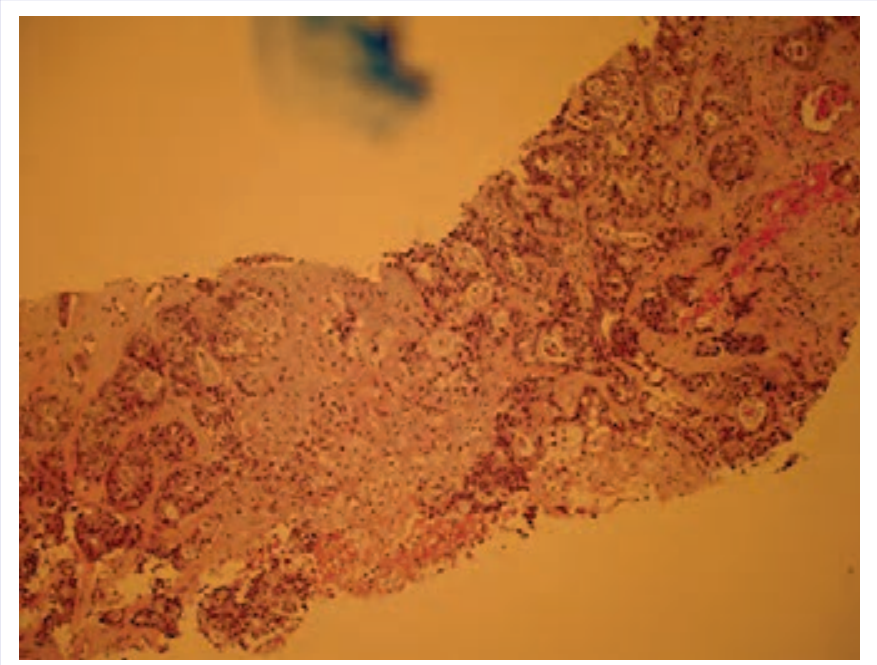

FIGURE 7 H\&E stain at 40x power showing metastatic adenocarcinoma in the liver. 
with a walking shoe. She then received whole brain irradiation treatment for her brain metastasis. After the whole brain irradiation treatment was completed, she was seen by medical oncology for chemotherapy options. Systemic chemotherapy was then considered, but the patient and her family decided to pursue hospice because of the progressive decline in her performance status. She succumbed to her cancer within 2 months of diagnosis.

\section{Discussion}

Acrometastasis as an initial presentation of metastatic non-small-cell lung cancer is an extremely rare finding. Osseous metastatic disease is found commonly in lung, prostate, kidney, breast, and thyroid cancers. Most commonly, acrometastatic lesions originate from primary lung cancers, specifically adenocarcinomas, followed by breast and renal-cell carcinomas. ${ }^{1}$ Despite their primary origin of tumor, they comprise only $0.1 \%$ of all osseous metastatic disease. ${ }^{2}$ Acrometastasis originating from a primary lung cancer will commonly have a presentation similar to that of diabetic ulcers or even osteomyelitis. They can present as painful lesions that are swollen and erythematous, and trauma may often be associated in the patient's history, as with the patient in this case. Acrometastasis can seem infected or even have small necrotic areas on the tips of the digit. There can be associated bleeding, weeping, or ulceration of the digit as well. ${ }^{3}$ Radiographic imaging should be obtained to help differentiate metastasis from osteomyelitis. In a case of metastatic disease, an MRI scan will show lytic lesions of the digit involved, with possible edema into the soft tissue in metastatic disease; but in a case of osteomyelitis, there will be evidence of abnormal marrow edema, soft tissue inflammation, and cortical bone destruction. ${ }^{4}$

More commonly, the digits of the fingers are more compromised with metastatic disease compared with digits of the foot. In the hand, the distal phalanges are the most common sites of metastatic deposits. ${ }^{2}$ Andreani and colleagues have reported that the most common site of acrometastases in the foot are the proximal locations, with tarsal bones being the most common at $50 \%$, followed by the calcaneus, metatarsal, and phalanx bones in decreasing frequency. ${ }^{5}$ Nicholson and colleagues have also described a case of cutaneous metastasis to a digit with ultimate bone invasion from the lesion. ${ }^{6}$ In that case, it arose from a squamous-cell lung carcinoma. This is generally not the case in metastases from adenocarcinoma. ${ }^{6}$

Initial presentation of an acrometastatic lesion to a digit is seldom seen in primary lung cancer, but it carries a very grim prognosis when it does occur. About 1 in 500 lung cancers with bone metastasis will be associated with digital metastasis. ${ }^{3}$ Even when a patient is diagnosed with acrometastasis to just a single digit, survival can be a short as 3-6 months from the time of original diagnosis. ${ }^{3}$ Treatment options are targeted toward palliation of pain and other symptoms, such as bleeding from the afflicted digit.

Radiation therapy can be given as a targeted treatment for local pain control. If possible, it should be offered within the first month of diagnosis or it may not be effective in the palliation of pain. Often there is advanced disease causing necrosis or osteomyelitis of the digit or joint that would require amputation. Most patients with severe pain from acrometastatis will have significant pain improvement after digit amputation.

After the patient has received targeted palliative treatment for the digit affected, systemic disease must be controlled. A chemotherapy regimen should be chosen based on the primary histology of the tumor and should be administered only if the patient has an adequate performance status.

Given the findings of the National Lung Screening Trial (NLST) with improved survival from lung cancer in screening with low-dose CT scans, our patient would have been an excellent candidate for surveillance screening with CT scan for detection of early lung cancer, possibly even in a curable stage. ${ }^{7}$ The NLST screened patients over a 3-year period at years 0,1 , and 2 with either a low-dose CT scan or chest radiography in patients with current smoking history of greater than 30 pack-years or who had quit smoking less than 15 years before. It excluded those who had hemoptysis, unintentional weight loss of more than 15 pounds, or had a CT scan in the 18 months before the baseline (year 0) screening. It compared low-dose CT scan with chest radiography for the early detection of asymptomatic lung cancer and mortality rates. Low-dose CT scanning detected 1,060 lung cancers, compared with 941 with chest radiography. In addition, low-dose $\mathrm{CT}$ scans detected more stage IA and IB lung cancers and fewer stage IV. There was a 20\% decrease in overall mortality from lung cancer in the CT scan group. Overall, the trial proved efficacious in highrisk patients for detecting asymptomatic lung cancers. ${ }^{\text {? }}$

About 7 million adults in the United States aged between 55 and 75 years have at least a 30 pack-year smoking history. Currently, the US Preventive Services Task Force gives a $\mathrm{B}$ recommendation for lung cancer screening in high-risk patients, that is, they recommend annual screening for asymptomatic adults aged 55-80 years who are actively smoking at least 30 pack-years, or who have quit within the last 15 years with low-dose CT scan for detection of early lung cancer. Screening should be discontinued when they have not smoked in more than 15 years, would no longer be a surgical candidate due to other comorbidities, or develop a life-threatening disease. There are other recommendations from the American Cancer Society and National Comprehensive Cancer Network, and the American Association for Thoracic Surgery also concurred with screening high-risk patients, although their inclusion criteria differ slightly. Smoking cessation coun- 
seling was mandated in all persons enrolled in the screening program because it remains the most important intervention to prevent lung cancer. ${ }^{8}$

Screening patients with low-dose CT scan does not come without some morbidity. False-positive scans can lead to overdiagnosis and invasive treatments, as well as possible psychological distress in some patients, although the latter was not formally reported. ${ }^{7}$ However, the effectiveness of early detection and treatment outweighed the potential pitfalls.

\section{References}

1. Spiteri V, Birba A, Ashwood N, Cobb J. Managing acrometastases treatment strategy with a case illustration. Ann R Coll Surg Engl. 2008;90:1-4.

2. Jakhar SL, Dana R, Punia DP. Cancer lung: an unusual presentation. Indian J Med Paediatric Oncol. 2009;30:149-150.

3. Long LS, Brickner L, Helfend L, Wong T, Kubota D. Lung cancer presenting as acrometastasis to the finger: a case report. Case Rep Med. http://dx.doi.org/10.1155/2010/234289. Published 2010. Accessed February 8, 2016.

4. Hochman M. Approach to imaging modalities in the setting of osteomyelitis. http://www.uptodate.com/contents/approach-toimaging-modalities-in-the-setting-of-suspected-osteomyelitis. Last updated August 23, 2012. Accessed February 8, 2016.
In summary, acrometastasis is an extremely rare finding in any malignancy. Once a patient has presented with acrometastasis, the prognosis is extremely poor with survival of only a few months. As with our patient, acrometastases represent a very advanced-stage cancer, and treatments are proposed and directed at palliation of symptoms to improve patient quality of life. If our patient had been compliant with annual care from her physician, she would have been an excellent candidate to screen for lung cancer given her long history of smoking.

5. Andreani L, Parchi PD, Piolanti N, Lisanti M. Finger foot phalanx metastases revealing occult pulmonary adenocarcinoma: a case report and review of the literature. Glob Adv Res J Med Medical Sciences. 2013;2:100-103.

6. Nicholson J, Mohamed I, Tahir A, Antunes G. Primary lung cancer presenting as metastasis to the big toe. J Surg Case Rep. 2011;12:5.

7. The National Lung Screening Trial Research Team. Reduced lungcancer mortality with low-dose computed tomographic screening. N Engl J Med. 2011;365:395-409.

8. Final Recommendation Statement: Lung Cancer: Screening. US Preventive Services Task Force. http://www.uspreventiveservicestaskforce.org/Page/Document/UpdateSummaryFinal/lung-cancerscreening . July 2013. Accessed February 12, 2016. 